\title{
Estimating the stability of a bed protection of a weir-mounted tidal turbine
}

\author{
Merel C. Verbeek, Robert Jan Labeur, Wim S. J. Uijttewaal
}

\begin{abstract}
Coastal infrastructure, such as bridges and storm surge barriers with weirs, provides an attractive location for harvesting renewable energy using tidal turbines. Often stone layers are applied downstream of coastal infrastructure to protect the sea bed from erosion. However, little is known about the potential effect of tidal energy extraction on the stability of this granular bed protection. This paper describes a study of the flow conditions influencing the stability of the bed protection downstream of a weir-mounted tidal turbine, using hydrodynamic data of an experimental test. The analysis indicates that the flow recirculation zone downstream of a weir may become shorter and flatter due to the presence of a horizontal-axis turbine. As a result, energetic turbulence eddies can transport more horizontal momentum towards the bed - hence the reason a heavier bed protection may be required for granular beds downstream of weirs when a turbine is installed. This information is essential when designing safe bed protections for coastal infrastructure with tidal turbines.
\end{abstract}

Keywords-Tidal turbine, Bed Stability, Turbulence, Experiments.

\section{INTRODUCTION}

$\mathrm{H}$ arvesting of renewable energy using tidal turbines is an attractive, but still expensive, sustainable alternative to conventional ways of energy production. The turbines can be installed in coastal infrastructure, such as bridges or open storm surge barriers, to bring down the costs compared to offshore deployments. Coastal structures constrict the tidal flow leading to relatively strong local currents - favouring a large energy yield and connect to the shore, facilitating a grid power connection. Often stone layers are applied downstream of coastal structures as erosion protection, but little is known about the potential effect of tidal energy extraction on the stability of this granular bed protection.

Since 2015, five horizontal-axis turbines (1.2 MW in total) have been operating in the storm surge barrier of the Eastern Scheldt tidal basin [1]. The barrier protects the South-Western part of the Netherlands from coastal

Manuscript received 16 March; accepted 26 March; published 12 May, 2020. This is an open access article distributed under the terms of the Creative Commons Attribution 4.0 licence (CC BY http://creativecommons.org/licenses/by/4.0/). This article has been subject to single-blind peer review by a minimum of two reviewers. The authors gratefully acknowledge Tocardo Solutions B.V. for the assistance in the test. flooding. The barrier consists of 62 neighbouring gates with weirs, connecting to a rock-fill sill construction. A granular bed protection is applied up to $600 \mathrm{~m}$ on both sides of the barrier (Fig. 1). A bed protection is needed as the flow velocity in the barrier is high - up to $5 \mathrm{~ms}^{-1}$ locally - and the flow is highly turbulent. The turbines are mounted in one opening of the barrier at one rotor diameter downstream of the concrete weir (Fig. 1). While the design of the bed protection of the barrier is conservative, the turbine influence is - strictly speaking not considered in the design calculations. Turbines may enhance the flow non-uniformity and increase the turbulence level of the flow, which may affect the stability of the stones of the bed protection.

Designs of bed protections are often based on vertical profiles of the streamwise flow velocity. The bed load and entrainment of stones cannot be predicted when using only near-bed flow velocities, since weirs induce large gradients in the velocity profile, with small or even negative near-bed velocities. Hoan et al. [2] and Hofland [3] even indicated that the bed load downstream of weirs is often caused by turbulent eddies in the water column.

The velocity gradients in the mean flow may result in turbulence organized in coherent fluctuations that can be schematized as an eddy. Fig. $2 c$ presents a schematization of a 2D turbulence eddy with a horizontal axis of rotation. The eddy moves with a mean velocity, $(\bar{u}, \bar{w})$, as it is passively transported with the flow. The lateral velocities can be neglected $\left(\bar{v} \approx 0, v^{\prime} \approx 0\right)$, assuming a laterally uniform flow. The turbulence motion of the eddy can both be described in terms of its horizontal length scale, $L_{H}$, indicating the size of the eddy, and in terms of the associated deviations from the mean velocity in the streamwise direction, $u^{\prime}$, and in the vertical direction, $w^{\prime}$. The eddy transports horizontal momentum towards the bed where it constitutes a bed shear stress.

The flow downstream of a hydraulic structure - a weir - may separate and recirculate downstream in a recirculation zone (Fig. 2a). The recirculation zone may be reduced in size in the presence of a turbine (Fig. 2b). The turbine redistributes the flow over the vertical, thereby

This work was supported by the Netherlands Organization for scientific research (NWO) within the programme The New Delta with project number 869.15 .008 and by the European Regional Development Fund (EFRO) OP-Zuid 2014-2020. M. C. Verbeek, R. J. Labeur, W. S. J. Uijttewaal are with Hydraulic Engineering Department, TU Delft Stevinweg 1, Delft, the Netherlands (e-mail: m.c.verbeek@tudelft.nl). Digital Object Identifier https://doi.org/10.36688/imej.3.21-24 


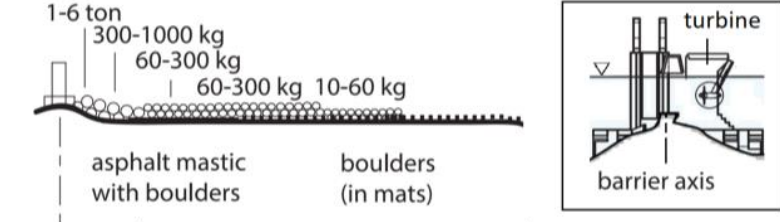

barrier axis $\quad$ bed protection $40-250 \mathrm{~m}$

Fig 1. A side view of the granular bed protection at the storm surge barrier in the Eastern Scheldt. The boulders are applied 40 to $250 \mathrm{~m}$ downstream of the barrier axis and weigh $10 \mathrm{~kg}$ to $300 \mathrm{~kg}$ each. The frame on the right details how the turbines are mounted relative to the barrier axis (weir). Adapted from Rijkswaterstaat.

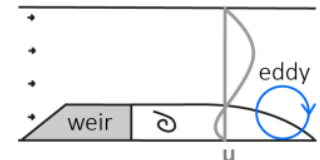

(a)
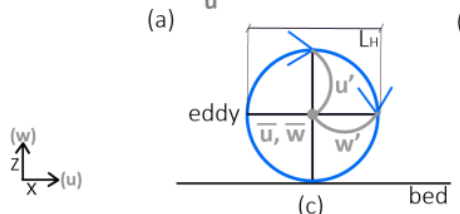

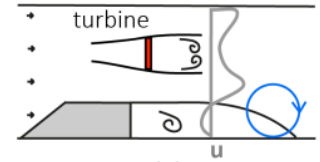

(b)
Fig. 2. A schematization (side view) of the non-uniform streamwise flow velocity profile downstream of a weir a), and downstream of a weir with a turbine b). Panel c) shows a 2D eddy structure with a horizontal axis of rotation which transports horizontal momentum towards to the bed, constituting a bed shear stress. LH is the horizontal eddy length scale. Adapted from [3].

changing characteristics of the $2 \mathrm{D}$ eddies in the flow locally. The turbulence parameters of the flow give insight in these eddy characteristics. The influence on the stability of the stones in a granular bed protection, in turn, may be estimated by adapting the stability parameter of the stones, using an engineering interpretation of the changed hydrodynamics [3].

Laboratory experiments can provide detailed hydrodynamic information in scaled situations that resemble the non-uniform flow downstream of a coastal structure with a tidal turbine. Information on the orientation and intensity of turbulence structures can be obtained, while this is not often available from field monitoring or numerical modelling of the flow. For example, no field measurements at the bed protection of the Eastern Scheldt could be conducted because of the harsh environmental conditions.

In this research, it is explored how the magnitude of the stability of a granular bed protection downstream of a weir changes when a turbine is installed, using turbulence and stability parameters to interpret the hydrodynamics. An experimental test, which closely resembles the flow past the weir of the storm surge barrier with turbines, is used to study the flow problem. This preliminary analysis is a first step towards designing safe bed protections downstream of turbines in barriers.

First, it will be described how to quantify the intensity and length scale of turbulence eddies and the resulting stability of a granular bed. Second, the set-up of the test, which provides the data for the analysis, is discussed. Third, the test data and calculated parameters are presented. Last, the bed stability downstream of a weirmounted tidal turbine is discussed.

\section{METHODS}

\section{A. Turbulence parameters}

Since the turbulence intensity - or turbulent kinetic energy - and the length scale of eddies in the flow govern the magnitude and duration of loads on the stones of a bed, they are estimated in the presented analysis. The flow is schematized with horizontal axis eddies and shear in the lateral is neglected, hence only the streamwise $u$ and vertical $w$ component of the velocity are used in the analysis.

The turbulence intensity, $I[-]$, is calculated as:

$$
I=U^{\prime} / \bar{U}
$$

where $\bar{U}$ is the mean velocity $\left[\mathrm{ms}^{-1}\right]$,

$$
\bar{U}=\sqrt{1 / 2\left(\bar{u}^{2}+\bar{w}^{2}\right)},
$$

and $U^{\prime}$ is the fluctuation from the mean velocity $\left[\mathrm{ms}^{-1}\right]$,

$$
U^{\prime}=\sqrt{1 / 3\left(\overline{\left.u^{\prime 2}+\overline{w^{\prime}}\right)}\right.}=\sqrt{2 / 3 k}
$$

which is proportional to the root of the turbulent kinetic energy $k$. In (2) and (3), $\bar{u}, \bar{w}$ denotes the time-mean velocity and $u^{\prime}, w^{\prime}$ the fluctuations from the mean (Reynolds decomposition). The fluctuation period of the eddies can be estimated with an integral time scale of turbulence. This time scale, $T$ [s], is defined as: [4]

$$
T=\int_{\tau=0}^{\tau_{1}} R_{u u}(\tau) d \tau,
$$

Where $R_{u u}$ is the autocorrelation function of the streamwise flow velocity [-], $\tau$ is a time lag [s], and $\tau_{1}$ is the time lag at which the autocorrelation equal zero. The autocorrelation function is defined as:

$$
R_{u u}(\tau)=\mathrm{E}\langle u(\tau)-\bar{u}, u(\mathrm{t}+\tau)-\bar{u}\rangle / \sigma_{u}^{2} .
$$

Here, $E$ is the expected value operator, $\sigma_{u}$ is the velocity standard deviation $\left[\mathrm{ms}^{-1}\right]$. The length scale, $L_{H},[\mathrm{~m}]$ or "size" of the eddy associated with $T$ [s] is calculated by multiplying the time scale with the local mean velocity, as is proposed in Taylors frozen turbulence hypothesis. [4] The bed shear stress, $\tau_{b}$, gives insight in the hydraulic load on the bed and is estimated as:

$$
\tau_{b}=\rho \overline{u^{\prime} w^{\prime}}
$$

Here, $\rho$ is the density of the water.

\section{B. Stability parameter}

The entrainment of stones from a bed protection can be estimated with a stability parameter for engineering purposes. A specific stability parameter is the Shields parameter, which gives the ratio between the eroding hydraulic load and the resisting gravitational load on a 
grain [6]. Shields considered a uniform streamwise velocity to estimate the hydraulic load. However, the flow downstream of hydraulic structures, such as weirs, has large gradients in the streamwise velocity over the vertical, which differs from the schematization of Shields. The stone stability can, in this case, only be predicted when including also information on the size and intensity of the turbulence in the water column [3].

Hofland [3] and Jongeling et al. [5] developed a stability parameter for this purpose, $\Psi[-]$, which gives the ratio between the quasi-steady hydraulic loads with loads due to turbulent eddies, and the resisting gravitational force,

$$
\Psi=\frac{\left(\max _{Z}\left[\langle\bar{u}+\alpha \sqrt{k}\rangle_{L_{H}} \frac{L_{H}}{z}\right]\right)^{2}}{\Delta g d} .
$$

The hydraulic load in the numerator of (7) can be calculated in four steps. At first, the temporal maximum of the instantaneous velocity, approximated with $\bar{u}+\alpha \sqrt{k}$ $\left[\mathrm{ms}^{-1}\right]$, is calculated for each streamwise and vertical position in the flow, since the largest velocities may affect the bed stability [3]. Here, $\alpha$ [-] is the empirical turbulence magnificator $(\alpha=5)$ [3]. Second, a moving average over a vertical distance $z \mp \frac{1}{2} L_{H}$ is taken, indicated with $\langle\ldots\rangle_{L_{H}}$, as an eddy influences the velocity surrounding its location up to a distance of half its length scale [3]. In this work, the turbulence integral length scale, $L_{H}$, is used to approximate the eddy length scale, rather than the Bahkmetev mixing length used in [3]. The former is preferred, as it incorporates the specific vertical structure of the flow at the weir. Third, the velocity estimate is weighted with the length scale of local turbulent structures in the flow, $L_{H}$ $[\mathrm{m}]$, relative to the vertical location of the structure, $z$, as only large scale eddies can reach the bed. The largest velocity estimate in the water column affects the stability at the bed, hence a maximum of the velocity estimate over the vertical is taken at last and fourth step [3].

The denominator of (7) gives the gravitational load and is composed of: $\Delta$, which is the specific submerged density of the stones of the bed protection $\left(\frac{\rho_{s}}{\rho}-1=1.6\right)[-], g$, which is the gravitational acceleration $\left[\mathrm{ms}^{-2}\right]$, and $d$, which is the stone diameter $[\mathrm{m}]$. The stone diameter is determined by the nominal diameter that is exceeded by $50 \%$ of the total mass of the stones. The bed is stable if the stability parameter is lower than the critical value, $\Psi_{-} \mathrm{c} \approx 1.2$ [3].

\section{Experimental set-up}

A series of experiments was conducted in the Environmental Fluid Mechanics Laboratory of Delft University of Technology with a horizontal-axis tidal turbine close to a broad-crested weir in a flume with a width of $0.78 \mathrm{~m}$, a depth of $0.85 \mathrm{~m}$, a streamwise length of $40 \mathrm{~m}$, and a flatbed to determine the flow turbulence and bed stability (Fig. 3). Two data series are used to explore the changed bed stability when a turbine is installed directly downstream of a weir. In the first series only a broad crested weir was installed in the flume, while in the

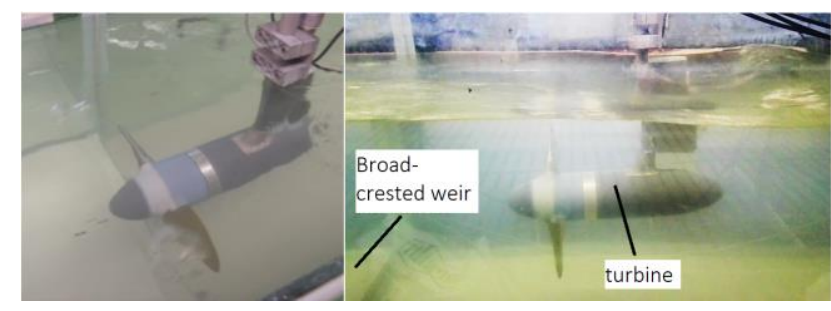

Fig. 3. The experimental set-up, consisting of a barrier weir with a two-bladed Tocardo Power Turbine (scale 1:9, rotor diameter $=0.4$ $\mathrm{m}$ ) in a flume of the Environmental Fluid Mechanics Laboratory of Delft University of Technology.

second series a weir and turbine were installed. During the tests, the discharge amounted to $0.430 \mathrm{~m}^{3} \mathrm{~s}^{-1}$ and the water level was $0.65 \mathrm{~m}$.

The dimensions of the model are defined using a Froude scaling of the flow at the weir of the Eastern Scheldt barrier, resulting in a scaled weir height of $0.05 \mathrm{~m}$, a 1:5 upstream slope and a $0.80 \mathrm{~m}$ long crest. The horizontal-axis turbine is geometrically scaled from the Tocardo Power Turbines T1, using the same ratio of 1:8.5 for model to prototype, respectively. The diameter of the rotor swept area (D) is $0.4 \mathrm{~m}$. The turbine tip speed is controlled with an Ac-servo motor and drive. The tip speed ratio is defined as the tip speed divided by the approach flow velocity.

The flow velocity is measured at each vertical and streamwise location with a 3-component side-looking and down-looking Acoustic Doppler Velocimeter (ADV, Nortek Vectrino). Three $3 \mathrm{~min}$ of data were collected per location with a sampling frequency of $25 \mathrm{~Hz}$ at an acoustic frequency of $10 \mathrm{MHz}$. The transducer sampling volume is $6 \mathrm{~mm}$ wide. A central system recorded water levels, turbine thrust, turbine torque, turbine tip speed and the flume discharge at $200 \mathrm{~Hz}$. The turbine Torque [Nm] and blade rotation frequency $\left[\mathrm{s}^{-1}\right]$ are measured in the turbine generator.

In the experiment no loads were measured at the flat flume bed. The velocity data, which was used to estimate the parameters that determine bed stability, was collected up to $3 \mathrm{~cm}$ from the bed. The bed in the flume was smooth, hence the flow field is not modified by the granular bed.

The power coefficient of the turbine, $C_{P}[-]$, is defined as:

$$
C_{P}=\frac{Q \omega}{\frac{1}{2} \rho u^{3} A_{0}}
$$

where $Q$ is the torque $[\mathrm{Nm}], \omega$ is the angular velocity $\left[\mathrm{s}^{-1}\right]$, $u$ is the undisturbed mean velocity at hub height, and $A_{0}$ is the rotor swept area $\left[\mathrm{m}^{2}\right]$. The power coefficient of the model amounts up to $40 \%$ of the value of the full scale prototype, which is sufficient to resemble the main physics in the scaled situation.

\section{RESULTS}

Fig. 4a presents the flow velocity in the flume in the two considered experiments: one with a broad crested weir and one with a weir and operating horizontal axis turbine. Both the weir and turbine increase the vertical non-uniformity of the flow by constricting the flow, resulting in locally 


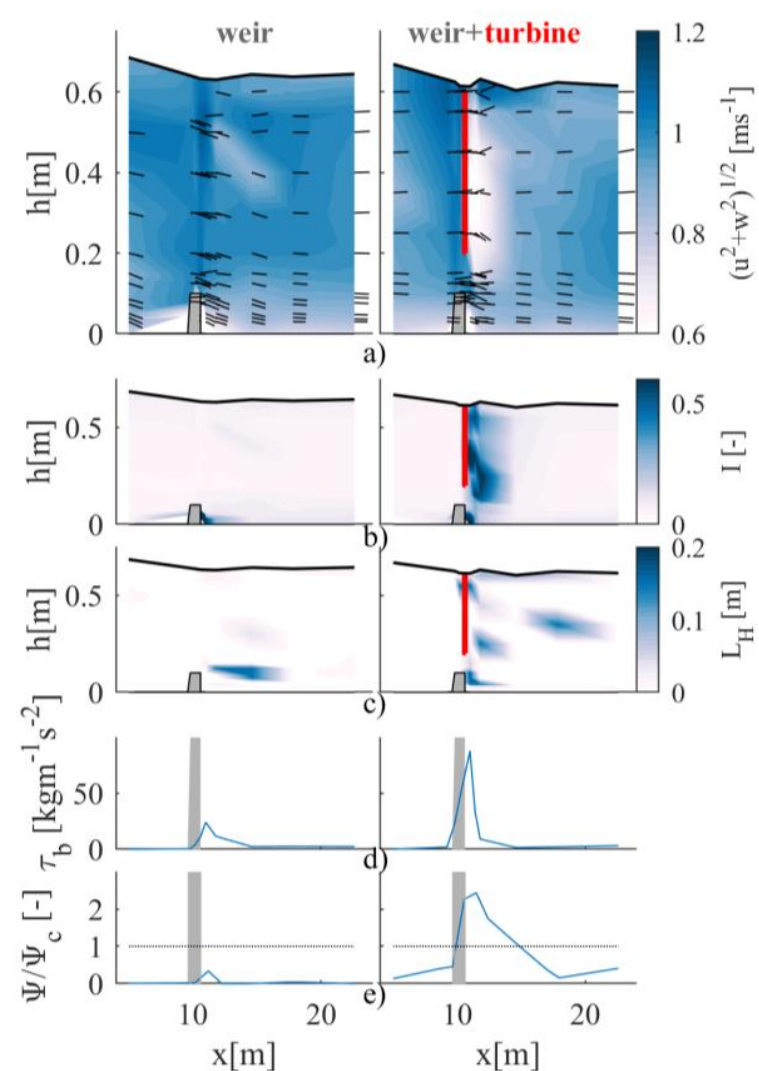

Fig. 4. Experimental results; a) The mean flow velocity along the centre cross-section of the flume for a situation with a broad-crested weir (left, weir in grey) and the situation with a weir and turbine (right, turbine in red), the small black lines are flow velocity vectors b) the turbulence intensity, I, for the same cross-section of the flume, c) turbulence integral length scale, $L H$, and d) the bed shear stress, $\tau_{b}=\rho \overline{u^{\prime} w^{\prime}}$, e) the ratio between the calculated stability parameter, $\Psi$, and the critical stability parameter, $\Psi_{c}$, at the bed (or at the top of the weir) for a $d$ of $1 \mathrm{~m}$. A higher ratio, $\Psi / \Psi_{c}$, implies a lower stability of the bed protection.

large flow velocities above the weir and below the turbine, the so-called bypass region. Besides, in presence of a turbine, a wake - a region with a velocity deficit - develops both downstream of the weir and downstream of the turbine.

The turbulence intensity and turbulence length scale are presented in Fig. $4 \mathrm{~b}$ and $4 \mathrm{c}$. The turbulence intensity is high in the shear layer surrounding the bypass flow, at the lower side of the turbine wake and at the upper side of the weir wake. The turbulence length scale, which is an estimate for the size of the eddies, is largest downstream of the weir. The eddy size downstream of the weir at crest height becomes smaller when the turbine is present, while eddies become larger in the lower part of the water column.

When a turbine is installed, the hydrodynamics at the bed change. While the bed shear stress increases only directly downstream of the weir crest, the stability parameter increases over a larger area downstream of the weir at the flume bed. The bed shear stress reflects only the increased turbulence intensity, while the stability parameter includes also information on the length scale of the flow structures.

\section{DISCUSSION}

The experimental data suggest that the bed stability directly downstream of a weir decreases when a turbine is installed. A number of processes may contribute to this decreased stability. When the turbine is present, the mean velocity and mean velocity shear near the bed increase. Additionally, more eddies with large length scale are present close to the bed downstream of the weir.

These changes are a result of a redistribution of the horizontal momentum of the flow downstream of the weir over the vertical. The bypass flow may constrict the wake downstream of the weir and cause the main flow to reattach at shorter distance from the weir. Hofland [3] and Stevens [7] showed that most damage to bed protections occurs in this area where the main flow attaches again to the bed downstream of a step in the bathymetry.

When a turbine is installed at a weir in situations with a granular bed protection, similar processes may influence the stone stability. As a consequence, a heavier bed protection may be required directly downstream of a weir. A possibility to overcome the decreased bed stability is to position the weir-mounted turbines closer to the water surface or further downstream of the weir to reduce the bypass flow below the turbine. However, the latter may reduce the power output of the turbine as well. In the situation of the Eastern Scheldt storm surge barrier the decreased stability of the bed protection should not cause any problems, as the stones are particularly heavy - a few tons of kilograms - close to the weir. At this location the bed protection is designed for more harsh conditions than the situation with a weir-mounted turbine.

This work showed a preliminary analysis of the stability of a bed protection downstream of a weir-mounted turbine. In future work the changed hydraulic load for different turbine operations, turbine positions, weir geometries and discharges will be evaluated.

\section{REFERENCES}

[1] M. C., Verbeek, R. J. Labeur, W. S. J., Uijttewaal, P., de Haas, "The near-wake of horizontal axis tidal turbines in a storm surge barrier", Proc. 12th EWTEC, Cork, Ireland, 2017, pp. 1179

[2] N. T, Hoan, M., Stive, R., Booij, B., Hofland, H. J., Verhagen, "Stability in Nonuniform Flow", J. Hydr. Eng., vol. 139, no. 9. Sep., 1, 2011, 10.1061/(ASCE)HY.1943-7900.0000387.

[3] B. Hofland, Rock and Roll, Turbulence-induced damage to granular bed protections, PhD Thesis, Delft University of Technology, 2005. Available: repository.tudelft.nl

[4] F. T. M. Nieuwstadt, B. J. Boersma, and J. Westerweel, "Correlation function and spectrum," in Turbulence - Introduction to theory and applications of turbulent flows. Springer Int., 2016, pp. 183-213. Available: springer.com/gb/book/9783319315973

[5] T.H.G., Jongeling, A., Blom, H. R. A., Jagers, C. Stolker, H. J., Verheij, "Design method granular protections." WLIDelft Hydraulics, Netherlands, Tech. rept. Q2933 / Q3018, Dec., 2003.

[6] A., Shields, "Anwendung der aehnlichkeitsmechanik und der turbulenzforschung auf die geschiebebewegung", Mitteilungen derPreussischenVersuchsanstalt fuer Wasserbau undSchiffbau, Berlin.

[7] T., Stevens. Student theses Delft University of Technology, The prediction of stone stability by a three-dimensional eddy resolving simulation technique, 2008. Available: repository.tudelft.nl 